\title{
Effects of COVID-19 Pandemic Response on Service Provision for Sexually Transmitted Infections, HIV, and Viral Hepatitis, England
}

Holly D. Mitchell, Tatiana Garcia Vilaplana, Sema Mandal, Natasha Ratna, Megan Glancy, Ammi Shah, Ruth Simmons, Celia Penman, Freja Kirsebom, Annastella Costella,

Alison E. Brown, Hamish Mohammed, Valerie Delpech, Katy Sinka, ${ }^{1}$ Gwenda Hughes, ${ }^{1}$ on behalf of the UK Health Security Agency National STI, HIV and Viral Hepatitis Surveillance Group ${ }^{2}$

Since the coronavirus disease pandemic response began in March 2020, tests, vaccinations, diagnoses, and treatment initiations for sexual health, HIV, and viral hepatitis in England have declined. The shift towards online and outreach services happened rapidly during 2020 and highlights the need to evaluate the effects of these strategies on health inequalities.

B eginning March 23, 2020, the UK government introduced social and physical distancing (SPD) measures to reduce transmission of severe acute respiratory syndrome 2 (SARS-CoV-2), and health staff were redeployed to the coronavirus disease (COVID-19) pandemic response. The staffing shift and SPD measures affected clinical services for sexually transmitted infections (STIs), HIV, and hepatitis $\mathrm{A}, \mathrm{B}$, and $\mathrm{C}(\mathrm{HAV}, \mathrm{HBV}$, and $\mathrm{HCV}$ ) provided through the National Health Service $(1,2)$. We assessed the effects of COVID-19 measures in England on service provision in this area and on health outcomes for persons with STIs, HIV, or hepatitis.

\section{The Study}

In England, surveillance of STIs, HIV, and hepatitis relies on patient-level data on consultations, tests, diagnoses, vaccinations, treatment, and outcomes from sexual health services (SHS), general practitioners, hospital outpatient clinics, and drug treatment centers (3). Laboratories also submit patient-level reports of tests and diagnoses for hepatitis and chlamydia. Given the disruption in routine reporting in 2020 (only 71\%-98\% complete for STI and HIV data), when

Author affiliation: UK Health Security Agency, London, UK

DOI: https://doi.org/10.3201/eid2803.211998 possible, we analyzed data from clinics and laboratories who provided complete reports for January-September in both 2019 and 2020.

Testing at SHS declined by $77 \%$, from 95,455 to 22,332 , for HIV and by $71 \%$, from 391,006 to 112,441 , for STIs during January-April 2020, and although there was a modest increase beginning in May, testing remained far lower than in 2019 (Figure 1). For January-September 2020 compared with the same period in 2019, overall numbers of tests were lower by $36 \%(768,216$ vs. 494,433$)$ for HIV and $28 \%(3,137,537$ vs. 2,244,153) for STIs (Appendix, https://wwwnc. cdc.gov/EID/article/28/3/21-1998-App1.pdf). However, the proportion of tests accessed through internet services (self-sampling kits returned directly to the laboratory with results provided by text message, email, letter, or online) increased substantially beginning in April 2020 (Appendix). Internet services accounted for $\geq 63 \%$ of HIV and $\geq 51 \%$ of STI tests during April-September 2020, compared with 25\% for HIV and 22\% for STIs in 2019.

During January-April 2020, the largest proportional declines in testing occurred among persons 15-19 years of age (79\% for HIV, 75\% for STIs) and $\geq 45$ years of age ( $80 \%$ for HIV, $76 \%$ for STIs); for persons 20-44 years of age, testing for HIV declined by $76 \%$ and for STIs by $70 \%$. The $15-19$ - and $\geq 45$-year age groups also showed the slowest relative recovery towards prepandemic levels of testing during June-September 2020. Over the same period, we observed larger proportional declines in testing among

${ }^{1}$ These senior authors contributed equally to this article.

${ }^{2}$ Additional group members who contributed are listed at the end of this article. 


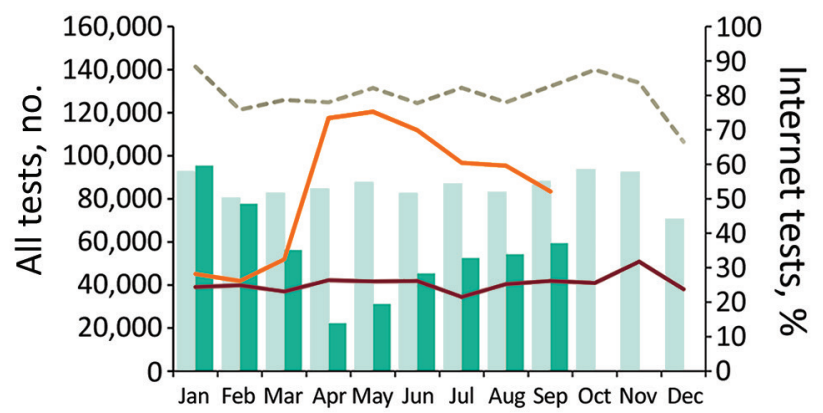

Figure 1. Total number of HIV tests provided through sexual health services (SHS) and proportion of those accessed through internet services, England, January 2019-September 2020. Bars compare HIV test data from SHS that reported complete data for JanuarySeptember in both 2019 (light green) and 2020 (dark green). Dashed line represents the total number of HIV tests from all SHS reported in each month in 2019. Solid lines indicate the percentages of total tests accessed through the internet for 2019 (red) and 2020 (orange). Data are from routine specialist and nonspecialist SHS reporting to the GUMCAD STI Surveillance System.

heterosexual men ( $81 \%$ for HIV, $79 \%$ for STIs) and heterosexual or bisexual women (women who have sex with men or women; $76 \%$ for HIV, $75 \%$ for STIs) compared with gay, bisexual, and other men who have sex with men (MSM) $(67 \%$ for $\mathrm{HIV}, 71 \%$ for STIs) and lesbian and other women who have sex exclusively with women (66\% for HIV, $65 \%$ for STIs); recovery was slowest among heterosexual men. We observed the largest declines among persons of Asian (81\% for HIV, 77\% for STIs), Black (81\% for HIV, 76\% for STIs), and other ( $81 \%$ for HIV , 76\% for STIs) races; persons of Black race showed the slowest recovery.

We also observed a sharp decline in the number of persons tested for hepatitis during January-April 2020: by $63 \%$ (from 4,295 to 1,610) for HAV, $61 \%$ (from 57,392 to 22,224 ) for $\mathrm{HBV}$, and $74 \%$ (from 43,238 to 11,250 ) for HCV (Appendix). The number of persons tested for $\mathrm{HCV}$ in community drug treatment facilities showed

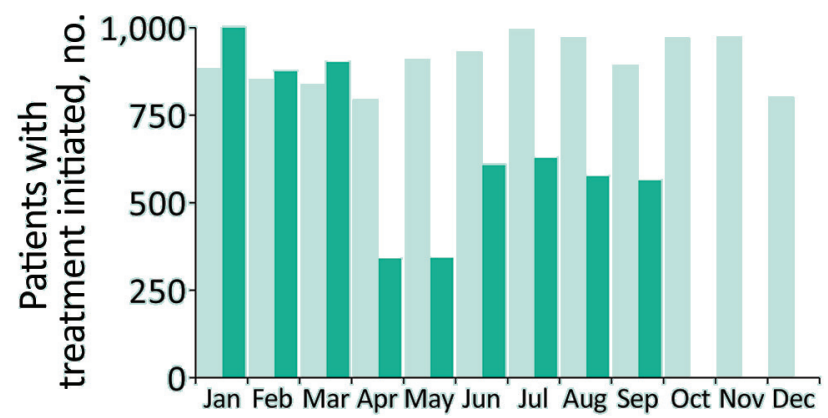

Figure 2. Hepatitis $C$ virus treatment initiations, England, January 2019-September 2020. Data are from the National Health Service England Hepatitis C Patient Registry and Treatment Outcome system. Bars indicate the number of persons having treatment initiated by month for 2019 (light green) and 2020 (dark green). the greatest decline (98\%, from 3,324 to 74 ) and a slow recovery to prepandemic levels; testing was $58 \%$ lower in September 2020 than for 2019 (3).

Consistent with testing patterns, the number of diagnoses for HIV, STIs, and hepatitis declined during January-April 2020, followed by a partial recovery (Appendix Figure 1). Bacterial STI positivity (Appendix) increased during March and April 2020 (12\% in January 2020 vs. 17\% in April 2020) then returned to 2019 and early 2020 levels, whereas HIV test positivity peaked in April $2020(0.20 \%)$ and remained at a higher level until September 2020 (0.09\% vs 0.10\% in September 2019). By contrast, HBV and HCV positivity declined during January-April 2020 (from 0.8\% to $0.4 \%$ for HBV surface antigen and from $2.8 \%$ to $1.4 \%$ for HCV antibody); although there was a slight increase thereafter, positivity remained lower for the rest of 2020 than in 2019.

The number of first-dose vaccinations administered to MSM at SHS during January-April 2020 fell by $97 \%$ for HAV (from 841 to 22 ) and human papillomavirus (from 1,507 to 47) and by $96 \%$ (from 757 to 34) for HBV (Appendix Figure 2). A slight increase was reported beginning in May 2020, but rates for $\mathrm{HAV}, \mathrm{HBV}$, and HPV vaccinations were $>50 \%$ lower in September 2020 than in September 2019.

$\mathrm{HCV}$ treatment initiations declined by $66 \%$ (from 1,004 to 341) during January-April 2020; although recovering slightly, the overall number of treatment initiations during January-September 2020 was 27\% lower than in the same period in 2019 (Figure 2). We saw the largest relative declines for referrals from drug services and prisons, with some recovery during June-September 2020. Delays commonly occur between HCV diagnoses and treatment, so reductions in treatment initiations likely reflected reduced access to services rather than new diagnoses alone.

\section{Conclusions}

The COVID-19 pandemic response in England, including the introduction of SPD measures, coincided with a decline in the provision of, and access to, health services for STIs, HIV, and hepatitis. We observed the greatest decline in services that cannot be provided remotely, such as vaccination.

These findings are supported by staff and peersupport surveys in SHS and community drug treatment services $(4,5)$. Some reduction in infections and need for services might be a consequence of reduced exposure because of compliance with SPD measures, leading to fewer opportunities for socializing and meeting sexual partners. The partial rebound in the summer of 2020 might indicate some recovery in 
service provision and demand, with increased demand also influenced by changes in risk perception and behaviors. However, these levels remained below prepandemic levels.

Declines in the numbers of STI, HIV, and hepatitis tests (6-8) and diagnoses (8-11) after COVID-19 restrictions began have also been reported across Europe and elsewhere. Disruption in HCV treatment provision is of concern as direct-acting antivirals clear the virus, minimizing long-term harms. HCV treatment disruptions have also been reported in Spain (12) and Germany (13); in Germany, a minority of treatment providers also reported an increase in delayed diagnoses of liver decompensation and hepatocellular carcinoma (13).

During the early stages of the pandemic, there was a rapid shift in service delivery toward online, remote, and outreach provision in England; similar shifts were reported in the United States and Croatia $(14,15)$. While enabling service access during the pandemic, it will be important to evaluate the effects on health inequalities of changing to remote services, because hepatitis, HIV, and STIs already disproportionately affect socially disadvantaged and excluded groups. Whereas our findings suggest SHS were accessed by some populations of need, such as MSM, the decline in access by young adults and persons of Asian, Black, or other races requires further investigation. Furthermore, early indications of adverse effects on access to harm reduction and bloodborne virus testing services for people who inject drugs is concerning and requires mitigating actions. The full effects of the COVID-19 pandemic response on STI, HIV, and hepatitis infection control, including efforts to eliminate HIV and hepatitis, and longer-term health outcomes will take time to emerge. These effects warrant close monitoring and assessment to ensure services are accessible and used by all who need them.

Additional members of the UK Health Security Agency National STI, HIV and Viral Hepatitis Surveillance Group who contributed to data collection, analysis and interpretation: Ana Harb, Galena Kuyumdzhieva, Tamilore Sonubi, Alireza Talebi, Stephen Duffell, Mateo Prochazka, Louise Thorn, Hannah Charles, Koye Balogun, Rebecca Wilkinson, Sara Croxford, Claire Edmundson, Mark McCall, Louise Logan, Adam Winter, Helen Harris, Kate Folkard, and Emily Phipps.

This work was conducted by the UK Health Security Agency (formerly Public Health England) as part of routine public health surveillance. No additional funds were received for this analysis.
No specific consent was required from the patients whose data were used in these analyses. The UK Health Security Agency has authority to handle patient data for public health monitoring and infection control under Regulation 3 of the Health Service (Control of Patient Information) Regulations 2002.

\section{About the Author}

Dr. Mitchell is an epidemiologist at the UK Health Security Agency. Her work focuses on surveillance and prevention of STIs and HIV.

\section{References}

1. Association of Directors of Public Health (UK). COVID-19 prioritisation of sexual and reproductive health service [cited 2020 Jul 21]. https:/ / www.adph.org.uk/2020/04/ covid-19-prioritisation-of-sexual-reproductive-health-services

2. Public Health England. People who inject drugs: HIV and viral hepatitis monitoring [cited 2020 July 28]. https://www. gov.uk/government/publications/people-who-inject-drugshiv-and-viral-hepatitis-monitoring.

3. Public Health England. COVID-19: impact on STIs, HIV and viral hepatitis [cited 2020 Dec 15]. https:/ / www.gov.uk/ government/publications/covid-19-impact-on-stis-hiv-andviral-hepatitis

4. British Association for Sexual Health and HIV. BASHH COVID-19 survey finds over half of services have been closed [cited 2020 Jul 21]. https:/ / www.bashh.org/news/ news/bashh-covid-19-survey-finds-over-half-of-serviceshave-been-closed

5. Action HCV; Public Health England. The impact of lockdown on hepatitis $C$ services in England. June 2020 [cited 2020 Nov 27]. http:/ / www.hcvaction.org.uk/sites/default/ files/HCV\%20Action\%20e-update\%20July\%202020.pdf

6. Pinto CN, Niles JK, Kaufman HW, Marlowe EM, Alagia DP, Chi G, et al. Impact of the COVID-19 pandemic on chlamydia and gonorrhea screening in the U.S. Am J Prev Med. 2021;61:386-93. https:// doi.org/10.1016/ j.amepre.2021.03.009

7. Simoes D, Stengaard AR, Combs L, Raben D. EuroTEST COVID-19 impact assessment consortium of partners. Impact of the COVID-19 pandemic on testing services for HIV, viral hepatitis and sexually transmitted infections in the WHO European region, March to August 2020. Euro Surveill. 2020;25:2001943. https:// doi.org/10.2807/1560-7917. ES.2020.25.47.2001943

8. Darcis G, Vaira D, Moutschen M. Impact of coronavirus pandemic and containment measures on HIV diagnosis. Epidemiol Infect. 2020;148:e185. https:/ / doi.org/10.1017/ S0950268820001867

9. Crane MA, Popovic A, Stolbach AI, Ghanem KG. Reporting of sexually transmitted infections during the COVID-19 pandemic. Sex Transm Infect. 2021;97:101-2. https://doi.org/10.1136/sextrans-2020-054805

10. Apalla Z, Lallas A, Mastraftsi S, Giannoukos A, Noukari D, Goula M, et al. Impact of COVID-19 pandemic on STIs in Greece. Sex Transm Infect. 2022;98:70. https://doi.org/10.1136/sextrans-2021-054965

11. Chia CC, Chao CM, Lai CC. Diagnoses of syphilis and HIV infection during the COVID-19 pandemic in Taiwan. Sex Transm Infect. 2021;97:319. https:/ / doi.org/10.1136/ sextrans-2020-054802 
12. Picchio CA, Valencia J, Doran J, Swan T, Pastor M, Martró E, et al. The impact of the COVID-19 pandemic on harm reduction services in Spain. Harm Reduct J. 2020;17:87. https:// doi.org/10.1186/s12954-020-00432-w

13. Hüppe D, Niederau C, Serfert $Y$, Hartmann $H$, Wedemeyer H; für das DHC-R. Problems in treating patients with chronic HCV infection due to the COVID-19 pandemic and during the lockdown phase in Germany [in German]. Z Gastroenterol. 2020;58:1182-5.

14. Bogdanic N, Javoric I, Skugor SB, Zekan S, Lukas D, Benkovic I, et al. Initial impact of COVID-19 epidemic on HIV services in Croatia. Infektol Glas. 2020;40:79-80.
15. Melendez JH, Hamill MM, Armington GS, Gaydos CA, Manabe YC. Home-based testing for sexually transmitted infections: leveraging online resources during the COVID-19 pandemic. Sex Transm Dis. 2021;48:e8-10. https://doi.org/10.1097/ OLQ.0000000000001309

Address for correspondence: Holly D. Mitchell, Blood Safety, Hepatitis, STI and HIV Division, Clinical and Public Health Group, UK Health Security Agency, 61 Colindale Ave, London NW9 5EQ, UK; email: holly.mitchell@phe.gov.uk
Middle East Respiratory Syndrome Coronavirus Transmission

- Acute Toxoplasmosis among Canadian Deer Hunters Associated with Consumption of Undercooked Deer

- Public Health Program for Decreasing Risk for Ebola Virus Disease Resurgence from Survivors of the 2013-2016 Outbreak, Guinea

- Characteristics of Patients with Acute Flaccid Myelitis, United States, 2015-2018

- Illness Severity in Hospitalized Influenza Patients by Virus Type and Subtype, Spain, 2010-2017

- Exposure to Ebola Virus and Risk for Infection with Malaria Parasites, Rural Gabon

- Cost-effectiveness of Screening Program for Chronic Q Fever, the Netherlands

- Unique Clindamycin-Resistant Clostridioides difficile Strain Related to Fluoroquinolone-Resistant Epidemic BI/RT027 Strain

- Porcine Deltacoronavirus Infection and Transmission in Poultry, United States

- Chronic Human Pegivirus 2 without Hepatitis C Virus Co-infection

- Interspecies Transmission of Reassortant Swine Influenza A Virus Containing Genes from Swine Influenza $A(\mathrm{H} 1 \mathrm{~N} 1)$ pdm09 and $A(H 1 N 2)$ Viruses

- Neutralizing Antibodies against Enteroviruses in Patients with Hand, Foot and Mouth Disease

\section{February 2020}

\section{Coronavirus}

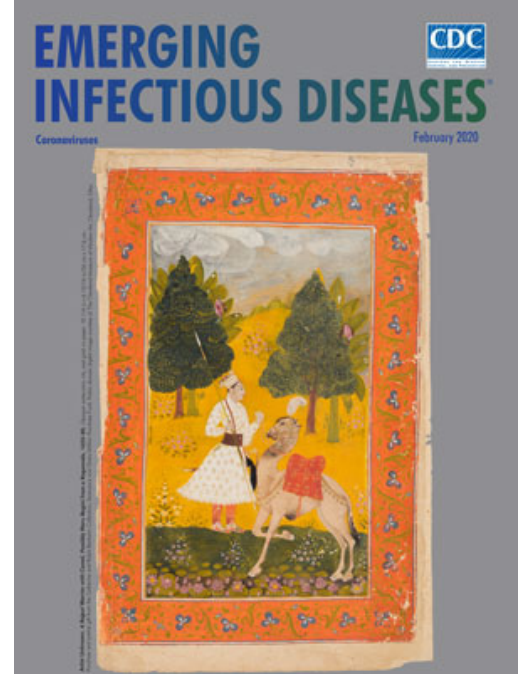

- Multiplex Mediator Displacement Loop-Mediated Isothermal Amplification for Detection of Treponema pallidum and Haemophilus ducreyi

- Novel Subclone of CarbapenemResistant Klebsiella pneumoniae Sequence Type 11 with Enhanced Virulence and Transmissibility, China

- Influence of Rainfall on Leptospira Infection and Disease in a Tropical Urban Setting, Brazil

- Systematic Hospital-Based Travel Screening to Assess Exposure to Zika Virus

- Rapid Nanopore Whole-Genome Sequencing for Anthrax Emergency Preparedness
- Elizabethkingia anophelis Infection in Infants, Cambodia, 2012-2018

- Global Expansion of Pacific Northwest Vibrio parahaemolyticus Sequence Type 36

- Surge in Anaplasmosis Cases in Maine, USA, 2013-2017

- Emergence of Chikungunya Virus, Pakistan, 2016-2017

- Mycoplasma genitalium Antimicrobial Resistance in Community and Sexual Health Clinic Patients, Auckland, New Zealand

- Early Detection of Public Health Emergencies of International Concern through Undiagnosed Disease Reports in ProMED-Mail

- Ocular Spiroplasma ixodetis in Newborns, France

- Hepatitis E Virus in Pigs from Slaughterhouses, United States, 2017-2019

- Rickettsia mongolitimonae Encephalitis, Southern France, 2018

- Human Alveolar Echinococcosis, Croatia

- Two Cases of Newly Characterized Neisseria Species, Brazil

- Hepatitis A Virus Genotype IB Outbreak among Internally Displaced Persons, Syria

- Rickettsia parkeri and Candidatus Rickettsia andeanae in Amblyomma maculatum Group Ticks

- Astrovirus in White-Tailed Deer, United States, 2018
EMERGING INFECTIOUS DISEASES
To revisit the February 2020 issue, go to:

https://wwwnc.cdc.gov/eid/articles/issue/26/2/table-of-contents 